\title{
Understanding Patients' WOM of IT-Enabled Healthcare Service: A Case Study of Online Health Consultation
}

\author{
Yezheng Liu \\ Hefei University of \\ Technology \\ liuyezheng@hfut.edu.
}

$\underline{\mathrm{cn}}$

\author{
Xue Zhang \\ Hefei University of \\ Technology \\ sherryz@mail.hfut.e \\ du.cn
}

\section{Abstract}

In healthcare context, the service delivery and information management are facilitated via varieties of IT-Enabled collaborative platforms. The effect of IT-Enabled collaboration health service has been extensively explored in recent decades. However, few studies have investigated the IT-Enabled health service from the perspective of patients or health consumers. Our research fills this research gap through a case study of an online health consultation website. The review systems on the website enable patients to write their word of mouth about services and physicians, thus providing us with a material to understand the interests, motivations and expectations of patients. In this work, we utilize four text-analysis techniques: term frequency analysis, sentiment analysis, feature words clustering and topic modelling to mining information from the textual WOM of patients. The results of our research have both theoretical and practical implications for optimizing the IT-Enabled health service, maintaining a harmonious physician-patients' relationship and increasing patients'satisfaction.

\section{Introduction}

Heath issue is usually challenging and costly. The government, caregivers and patients are actively exploring methods to control the cost of health care through the evolution of medical technology, the innovation of care delivery channel, the collaboration of healthcare resources and the application of patients management systems[24]. Understandably, patients traditionally turn to physicians, family members or friends for medical or emotional support to deal with their health issues [20]. However, due to the insufficient healthcare resources in developing countries, there are amounts of rural patients have no effective access to healthcare service [12]. In recent decades, the development of information technology brings opportunities to facilitate the healthcare delivery in developing areas. In particular, a fast growing number of telemedicine programs have been established around the world [25]. The concept of telemedicine emerged approximately four decades ago, basically devoted to promote the collaboration among health professionals and technologists via telecommunications and bio-medicine technology to support remote patients care or health service collaboration [24]. Currently, as the advance of a variety of Internet and communication technologies (ICT), the flexibility and accessibility of online resources attract more and more patients to turn to the Internet to seek information, healthcare service and social support [32] as they played an energetic role in the process of making decisions about their health care and treatment. For example, the search engine has been commonly used to search for information support about health concerns [40]. Similarly, the social network and online communities attract more and more patients to communicate about their health issues [42]. Specifically, the widespread adoption of online health communities (OHCs) make a great difference in the transformation of the experiences of healthcare such as peer-to-peer support, chronic disease management and information collaboration among patients, caregivers, health professionals, and policy makers [21]. In the early stage of $\mathrm{OHC}$, its participants are usually health consumers. Through engaging in health issue related discussion online, they gain external information support and emotional support from similar patients [44]. Now, the participation of health professionals extend the schema of online health communities by means of providing online health consultation, clarifications, explanations, as well as possible reference, resources, and opinions about medical issues, remedies, and therapies for patients and other health consumers [18]. As a consequence, websites or forums providing 'e-Health services' emerge one after another. In China, there are some famous online health websites, for example, the Haodf.com (http://www.haodf.com/) 
and the Guahao.com (http://www.guahao.com/). Those websites utilize interactive communication and information technology to bridge the gap between healthcare consumers and caregivers. Through enhancing the physician-patient relationship and providing access to valued resources that can be shared among members, they advocate the information and service collaboration among health professionals, researchers, patients, and their family members, with the goal of tackling health issues [2]. To date, there have been a large number of studies and systematic literature reviews on IT-Enabled collaboration in healthcare context, ranging from the design of health information systems [6] to the implementation of HIT [36]. Despite previous research, very few studies investigate the users' WOM of the IT-Enabled collaborative health service delivery. As is a consensus that understanding users' needs is fundamental for the design of information systems, designing effective online health platforms using ICT also requires in-depth understanding of users' health service needs. Based on such principle, we propose our main research question:

RQ: What is the user's WOM of the IT-Enabled healthcare service?

To address these issues, we take a famous online health consultation website as a sample to study users' online WOM about IT-Enabled healthcare service. Understandably, users' WOM expressed in natural language are a comparatively rich reflection of their positive attitude and real demand. Through using text analysis method, this study intends to analyze characteristics of user-generated posted reviews after counseling a health professional. Our research contributes to both the research and practice of IT-Enabled collaborative healthcare service, through extending the application of text analysis method in understanding patients' attitude. The case study reveals patients' interest, demand and motivations when using IT-Enabled health service, thus providing practical implications for care providers, policy makers and website designers.

This paper is structured as follows. First, IT-Enabled collaboration in healthcare, online WOM and text analysis method are reviewed. Second, the research context including dataset and method is presented. Result from the case study are presented and explained, followed by the conclusion and discussion describing users' WOM on IT-Enabled healthcare service. Finally, the managerial implications and limitations of the research and suggestions for future research are given.

\section{Literature Review}

\subsection{IT-Enabled collaboration in healthcare and online health consultation}

With the growth of information technology, the IT-Enabled collaboration emerged a few decades ago, and has been commonly applied in many field, such as education, work-flow, industry and logistics [1]. Previous study has confirmed the effectiveness of IT-Enabled collaborative method. To balance the unmet healthcare demand and the shortage of healthcare providers and medical resources, government agencies and foundations have increased financial and human resources investment to collaborative projects for health research and education[21], such as telemedicine and Electronic Health Record(EHR). These practices not only facilitate the medical resource allocation and collaboration between developing areas and developed areas, but also allow remote patients to access medical support such as pre-diagnosis and treatment, through the Internet, via computers or wireless devices. In recent years, advances in information and communication technologies (ICT) have raised health consumers' expectations for health. There is an obvious tendency that more and more patients participate in health information seeking, self-management and health decision making. Accordingly, the concepts of Health 2.0 and Medicine 2.0 have been introduced [43]. In this context, ICT offers a promising means to establish unparalleled and direct collaborative interaction between healthcare professionals and patients. As a typical application of IT-Enabled collaboration in healthcare, the online health consultation service provides alternatives to face-to-face consultations in virtue of social media, telephone and live interactive video. Through online health consultation, doctors could not only get money reward but also obtain the social reward, for example, get higher reputation and, improve patient flow [45]. For patients, online health consultation serves as an alternative source of medical support to cope with their illness. This interactive service allows patients to ask questions related to their disease and receive customized therapy based on personal health conditions and disease severity [29]. Although these services are increasing in number, they need to be evaluated for their potential to provide convenient and efficient care for specialty services.

\subsection{Online Word-of-Mouth}

People often publish their WOM to express their comments on a service or a product. While traditional WOM usually consists of spoken words and exchanged with familiars in a face-to-face situation, online WOM 
involves personal experiences and opinions transmitted through the written word [41]. The online WOM are popular among electronic commerce website, allowing users' write down their reviews or judge a product based on previous reviews [27].

There have been many researches investigating the effect and of users' WOM. For example, in marketplace, lots of researchers have found that online WOM significantly impacts consumers' consumption behaviors [37], product sales performance [10] and reputation [7]. In service industry, users WOM or reviews also play an important role to influence experiencers decision making such as service evaluation, channel selection [28]. In the IT-Enabled healthcare platforms, WOM are also necessary to help users share their service experience, rank physicians service quality and express satisfaction. Previous studies have devoted much attention to the numeric ratings of physicians have received broad attention from both the media and other physicians [31]. Specifically, researchers have explored the technical ratings and functional ratings of WOM on patients' choice [30]. Compared to numeric ratings, the textual comments could be a more vivid reflection of users' real mind, thus would be an important resource to understand users. However, currently there is a lack of investigation on textual WOM in the context of IT-Enabled healthcare.

\subsection{Text analysis method in healthcare context}

Text mining techniques have been developed to extract and quantify the textual information and useful knowledge from textual corpus [26]. As it is known, there are some classical text analysis method, which is effective, accurate and generalized in dealing with textual content. For example, the topic modelling and sentiment analysis. are commonly used to mining users opinion and calculate users' emotion valence [34], respectively. Compared to the human-annotated method, these automatic text mining methods are much more efficient and low-cost. Hence, more recently, text mining techniques have been adopted by medical fields, such as extracting terms from clinical text [17], analyzing medical research publications to match domain ontology [9] and investigating the effect of sentiment features in locating the online mentions of adverse drug reactions [23]. Specifically, through studying users' online posts (i.e. textual reviews), we can understand patients opinions [15] (i.e. Positive, negative or neutral), emotions (i.e. sad, anxiety) [33] and demands, by means of sentiment analysis. In our study, patients online WOM are written as textual content, therefore, the text analysis method plays an essential role in mining the characteristics of the textual WOM. In this way, we are able to analyze the patients' attitude, needs and motivations.

\section{Research context}

\subsection{Dataset}

3.1.1. Online health consultation website background. Like other OHCs website, Guahao.com provide health information inquiry, health consultation and online appointment services. Physicians are divided into specific board based on their departments or forte or hospital. Patients could explore information about the physicians through checking description information provided by themselves, or by referring to other patients WOM.

3.1.2. Data collection and preprocessing. To obtain the secondary data, we set up Java WebCrawler which started on March 8th,2018 to crawl patients' reviews, the crawler lasted for two days. We obtain 384,650 reviews for 3,588 physicians, with an average of roughly 107 reviews per physician. Raw corpus are made of long sentences with the combination of words, some also including nonsensical symbols, punctuations, numbers and stop words. These meaningless elements are called as noise and will increase the difficulty of text analysis and influence the analysis accuracy, for example, the stop words like "is" and "the" will occupy most of the frequency thus reducing the real frequency of special words. To address this issue, a preprocess procedure is conduct for all reviews before further analysis. As is shown in Figure 1. The preprocessing procedure followed by data crawling and storing, contains segmentation and filtering (to remove stop words, numbers and punctuation).

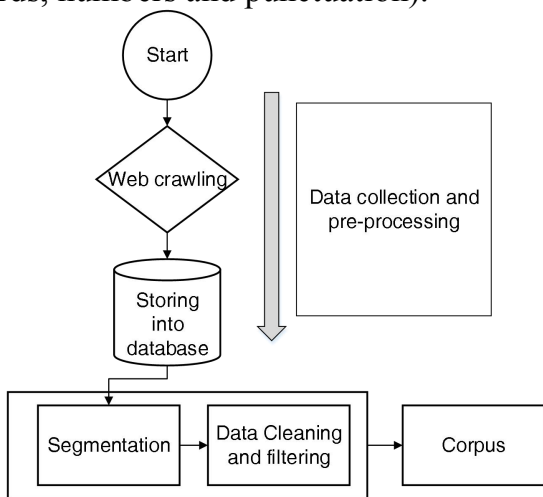

Figure 1. Data collection and pre-processing

\subsection{Bigrams frequency analysis}

Though count the term frequency in a document, 
we are able to make a good sense of the main concerning of the publisher. Accordingly, we utilize the widespread used TF and TF-IDF methods to calculate the important referring in patients WOM.

3.2.1. TF Frequency. For given text documents, the number of times a term occurs in a document is called its term frequency. In general, term frequency refers to the most relevant information included in the document. Given a document $\mathrm{d}$, the term frequency of term $t, t f(t, d)$ is defined as follows:

$$
t f(t, d)=f_{t, d}
$$

Where $f_{t, d}$ equals the number of times that term $\mathrm{t}$ appears in document $d$. For document set $D$, the total frequency of term $t$, is defined as:

$$
t f(t, D)=\sum_{d=1}^{D} f_{t, d}
$$

Where $d$ is an individual document in $D$.

\subsubsection{Term Frequency-Inverse Document}

Frequency (TF-IDF). In the case of using raw count of a term as the term frequency could tend to incorrectly emphasize some common words more frequently, for example, the word 'physician' may occur in most of the patients' reviews. However, this term delivers limited discriminative information, without giving enough weight to the more meaningful terms.

Therefore, an inverse document frequency factor is introduced which diminishes the weight of terms that occur very frequently in the document set and increases the weight of terms that rarely occur [39]. Actually, TF-IDF count term frequency through determining the relative frequency of words in a specific document compared to the inverse percentage of that word over the entire document set [46]. Given a document $\mathrm{d}$, which included in document set $\mathrm{D}$, the TF-IDF of term $t$ can be calculated as follows:

$$
\operatorname{tfidf}(t, d, D)=t f(t, d) \times i d f(t, D)
$$

\subsection{Lexicon-Based sentiment analysis}

The lexicon-based approach generally relies on a series of standard dictionaries such as LIWC to map words within corpus and compute its sentiment or determine the sentiment polarity [11]. Table 1 summarize dictionaries used in this paper. Those standard dictionaries contain sentiment words (i.e. positive words and negative words) dictionary, degree words dictionary (i.e. very, most) and negative words dictionary (i.e. not, no).

As shown in Figure 3, the documents would be segmented into pieces, then stop words, punctuation and numbers would be removed. It is a necessary procedure to filter noisy terms. Finally, to do sentiment analysis, the 'clean' corpus would be mapped with each standard dictionary. For the corpus $C$, its sentiment can be computed as follows:

$$
\operatorname{sentiment}(C)=\sum_{w=1}^{W}(-1)^{p} \times d \times S_{w}
$$

Where $W$ denotes the number of clauses in corpus and $w$ is a single clause, $S_{w}$ denotes the original standard sentiment score of the keyword in $w, p$ represents the number of negative words that embellish $w$, and $d$ appends the sentiment degree of $S_{w}$ according to the degree word dictionary. Exclusively, if $p$ is odd, the sentiment of $w$ would be inverted.

Table 1. Standard sentiment dictionary

\begin{tabular}{|c|c|}
\hline Category & example \\
\hline $\begin{array}{c}\text { Ontology } \\
\text { dictionary }\end{array}$ & $\begin{array}{c}\text { surgery, treatment, cardiopathy, } \\
\text { diabetes }\end{array}$ \\
\hline $\begin{array}{c}\text { Sentiment } \\
\text { dictionary }\end{array}$ & negative: bad, terrible, dislike \\
\cline { 2 - 2 } & positive: good, like, professional \\
\hline $\begin{array}{c}\text { Degree word } \\
\text { dictionary }\end{array}$ & $\begin{array}{c}\text { most degree, very degree, more } \\
\text { degree, general degree, least degree }\end{array}$ \\
\hline $\begin{array}{c}\text { Privative word } \\
\text { dictionary }\end{array}$ & no, not, none \\
\hline
\end{tabular}

\subsection{Feature words analysis}

In the present work, we employed a text analysis program called TextMind to cluster feature words in patients' reviews. TextMind is a Chinese language psychological analysis system developed by Computational Cyber-Psychology Lab, Institute of Psychology, Chinese Academy of Sciences. TextMind provides easy access to analysis of the preferences and degrees of different categories in a text. Inspired by the dictionary of LIWC2007 and C-LIWC, TextMind is developed based on the characteristics of Simplified Chinese language in mainland China [14]. The clustered words categories could reveal the user's attention focus, thinking styles and individual differences in language use.

\subsection{Topic model analysis}

Topics generally refer to the hidden structure in an article, blogs or review. Topic models are algorithms developed for discovering the focal hidden themes that pervade a huge and other unstructured collection of documents [4]. In this paper, we use latent Dirichlet allocation (LDA) [5] to mining topics in patient reviews. As one of the most elementary topic models, LDA has been widely used in topic mining and text clustering. For each document $w$ in a corpus $D$, the 
LDA process can be described as:

(1) Select $N$ words from $w$, where $N \sim \operatorname{Poisson}(\xi)$;

(2) Select $\theta$, where $\theta \sim \operatorname{Dir}(\alpha)$;

(3) For each of the $N$ words $w_{n}$ :

Select a topic $z_{n} \sim \operatorname{Mutinomial}(\theta)$;

Select a word $w_{n}$ from $p\left(w_{n} \mid z_{n}, \beta\right)$, a multinomial probability conditioned on the topic $\mathrm{z}_{\mathrm{n}}$.

The algorithm mechanism can be defined as the following graph:

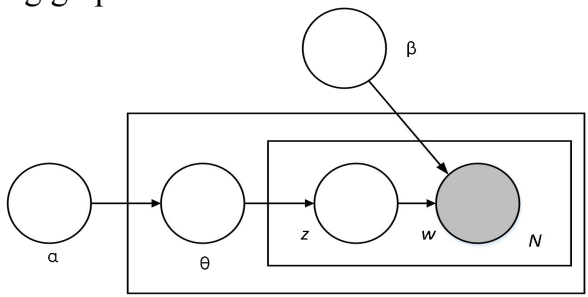

Figure 2. Graphical model representation of LDA

Posterior computation is a crucial issue of topic modelling. Generally, there are two kinds of computational approaches: sampling-based algorithms and variational algorithms. In our present work, We introduce Gibbs sampling [16] schemes to speed up the parameter estimation. Choosing the proper number of topics is another problem in topic discovery. To deal with this problem, we set up an iterated modelling method, we set a range of topic numbers $K=\{5,10,20$, $30,40,50,60,70,80,90,100\}$, for each $\mathrm{k}$ in $\mathrm{K}$, there will be a ten-fold training procedure. Then we could obtain a perplexity index and a log-likelihood index for each fold. The perplexity is a conventional index to evaluate the performance in language modelling, is monotonically decreasing in the likelihood of the test data, and is known as the exponential of the average negative log-likelihood [3]. A lower perplexity score indicates better generalization performance. Oppositely, A higher likelihood score suggests better modelling ability.

\section{Research results}

\subsection{Descriptive statistics analysis}

Through web crawling, we finally obtain 384,650 reviews for 3,588 doctors. Those reviews were posted between June, 14th 2012 and March, 9th 2018. Figure 5 shows that there was a moderate increasing from 2012 to 2015 , while a sharp upward tendency occurs in 2016.

To our knowledge, Guahao.com accomplished its biggest financing on September, 2015, from then on, it made massive progress in the Internet healthcare industry and attracted more and more users, including patients and physicians joining in Guahao.com.

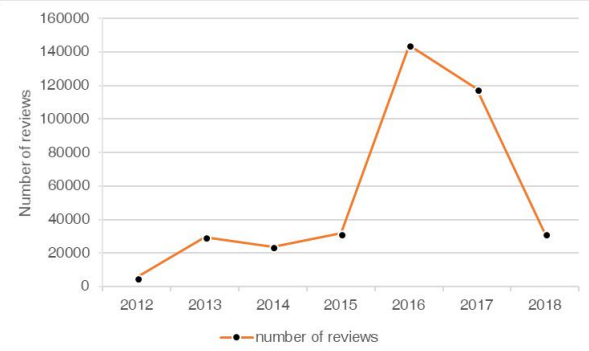

Figure 3. Number of reviews from 2012-2018

From Table 2, we can find that some physicians got more reviews than others, for a reason is that those physicians may provide higher quality services or hold better reputation, thus attract more patients.

Table 2. Number of reviews of physicians

\begin{tabular}{|c|c|}
\hline Number of reviews & Number of physicians \\
\hline$<10$ & 453 \\
\hline $10 \sim 50$ & 1543 \\
\hline $50-100$ & 584 \\
\hline $100-200$ & 422 \\
\hline $200-500$ & 364 \\
\hline$\geq 500$ & 222 \\
\hline
\end{tabular}

For further statistical analysis, as shown in Figure 4, patients usually wrote reviews between 9:00 am and 19:00 pm. Interestingly, there was a peak at 20:00 pm. It could be a signal that people are usually free during this time, and care more about health issue than any other period.

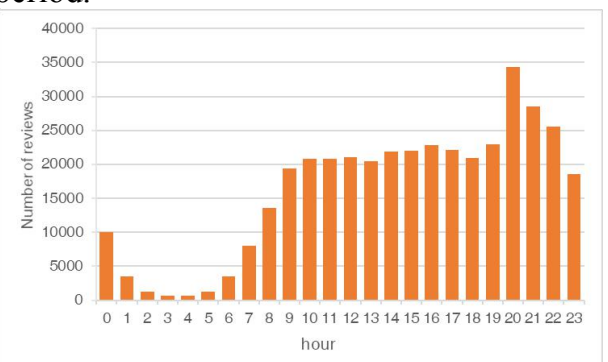

Figure 4. Number of reviews on different hours

\subsection{Text analysis results}

4.2.1. Frequent bigrams distribution. Figure 5 indicates the top 20 frequent terms in patients' reviews calculated by TF. Terms such as 'physician', 'thanks' and 'patient' show literally high occurrence rate. It is because that most of the reviews contain these words. As discussed in part 3.2, TF uses raw count of terms as frequency. Hence it is not helpful to find discriminative 
terms. As a consequence, we recompute the term frequency using TF-IDF.

As displayed in Table 3, the most frequent words changed and some special terms arisen, for example, 'environment', 'surgery' and 'appointment', these words may not be very frequent in the whole document set, but they capture discriminative information of the review. Through these words, we can find patients' focus attention, in other words, what the patient care about and what they want. Also, we can find that some terms may relate to patients compliant, for example, 'wait' and 'sad', patients would express their dissatisfaction about long waiting time and disappointing service quality through reviews. Hopefully, it will be helpful for other potential patients to adjust their consultation decision.

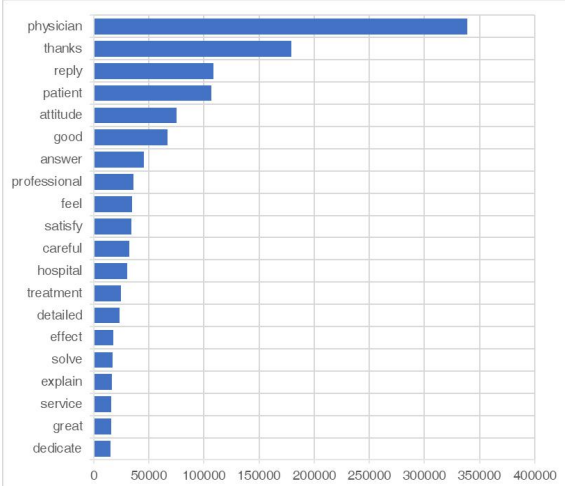

Figure 5. TF frequency of terms

Table 3. TF-IDF frequency of terms

\begin{tabular}{|c|c|}
\hline $\begin{array}{c}\text { TF-IDF } \\
\text { Frequency }\end{array}$ & Terms \\
\hline $20000-30000$ & reply, environment, treatment, effect \\
\hline $15000-20000$ & $\begin{array}{c}\text { feel, answer, hospital, patient, time, wait, } \\
\text { surgery, professor }\end{array}$ \\
\hline $13000-15000$ & $\begin{array}{c}\text { sad, appreciate, check, app, appointment, } \\
\text { flexible }\end{array}$ \\
\hline $12000-13000$ & great, solve, thanks, mood, photo, mobile \\
\hline $11000-12000$ & $\begin{array}{c}\text { question, record, Chinese-herb, therapy, } \\
\text { simple }\end{array}$ \\
\hline $10000-11000$ & $\begin{array}{c}\text { attitude, detail, careful, revisit, condition, } \\
\text { explain }\end{array}$ \\
\hline
\end{tabular}

4.2.2. Sentiment distribution. Figure 6(a), (b) and (c) display negative review rate, neutral review rate and positive review rate of 3,588 physicians. It is evident that positive reviews occupy the most proportion of reviews. The percentage of negative reviews mainly lie between $10 \%-15 \%$, the percentage of neutral reviews shows a similar distribution.

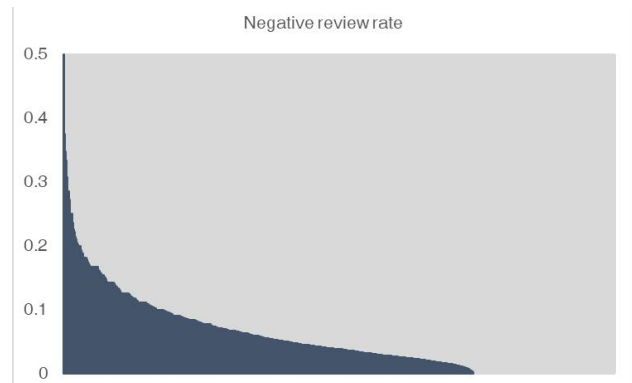

Figure 6(a). Negative review rate for each doctor

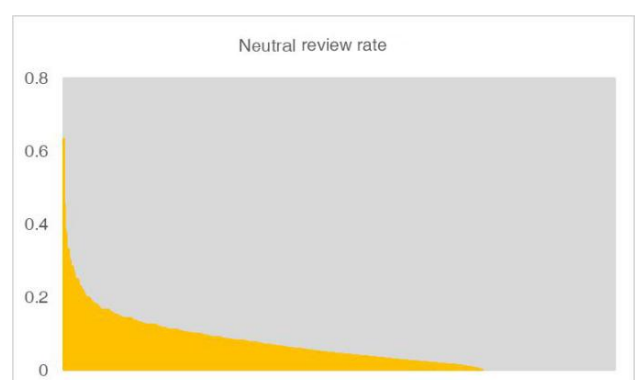

Figure 6(b). Neutral review rate for each doctor

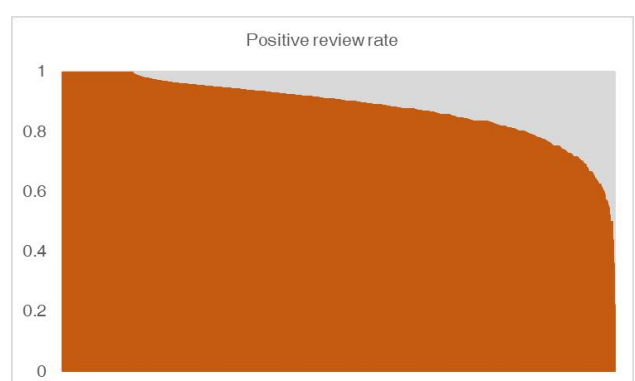

Figure 6(c). Positive review rate for each doctor

According to Figure 6(d), we find that the average sentiment score of per review of each physician mainly lies between +2 and +4 . It indicates that most of the reviews are written in a placid mood, in other words, only a few patients put lots of emotional expressions into the review.

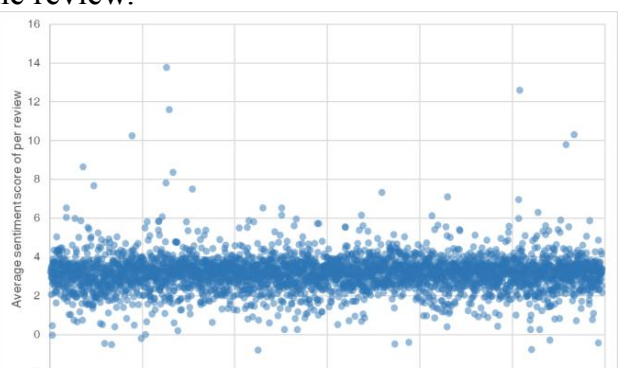

Figure 6(d). Average Sentiment score of reviews for each doctor

However, it should not be ignored that there are still some precisely negative reviews, which 
implicitly contain notable information represent that the service and website quality, doctor's literacy need to be improved. According to prior research, compared to positive information, negative information may have a significant impact on evaluations [19]. Studies also find that negative reviews tend to play a more meaningful role or serve as a reminder signal in the marketplace [8]. For further discussion, we have some samples in table 4 to illustrate what makes patients dissatisfied.

Table 4. Samples of negative reviews (1) Impatient! Bad attitude! Let me wait for so long but didn't reply!

(2) Received my money but did not deal with my consultation. No medical ethics!

(3) The doctor is totally irresponsible and the price is very high. I am not satisfied with his answer.

(4) The treatment plan is absolutely incorrect. It's so terrible.

(5) The description of the function is vague, too troublesome to initiate a consultation.

From Table 4, it is noted that patients would make complaints about waiting time, doctors' attitude as well as service quality and the function vulnerability of the website or mobile applications. Most of the negative reviews carry quite strong emotions, i.e. anger or disappointed. These reviews could be an alert for potential users of the service, remind them of possible loss of money and time. Meanwhile, it can be useful clue represent users' requirements, thus help the manager of the online health community as well as website developers to renovate their functionality and marketing strategy.

4.2.3. Feature words distribution. Figure 7 displays the clustering result of feature words within patient reviews.

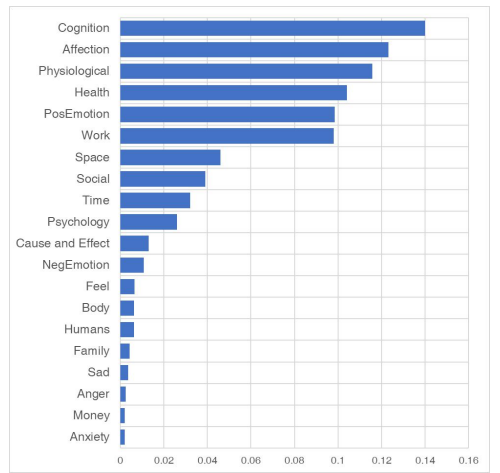

Figure 7. Feature words distribution

As we can see, the most frequent feature set is the words represent people's cognition, affection and physiological index. It is easy to understand this phenomenon since these reviews come from healthcare (or medical) fields. Users in online health communities tend to mention their health conditions, emotions and feeling because it can be a release of stress and anxiety [38], which is brought by poor health situations and pain of disease. Apart from that, words referring to work and social life are often mentioned, it is probably because people usually relate their health issue with work load and daily experience [47].

\subsection{Topic model result}

Through running the topic modelling algorithm, we obtain a series of perplexity index and likelihood index which represent the modelling performance under different topic numbers. According to Figure 8 and Figure 9, it is apparent that the most appropriate topic number approximate 50 . As a consequence, we launched LDA with a topic number of 50 . Then, each corpus is assigned to one or more topic, for illustration, we select five topics and exhibit the most frequently occurring words in each topic in Table 5.

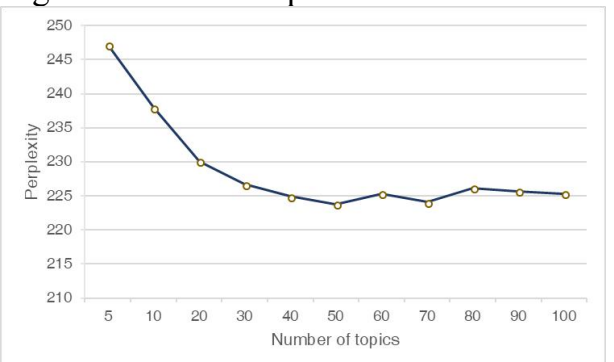

Figure 8. Perplexity trend of LDA

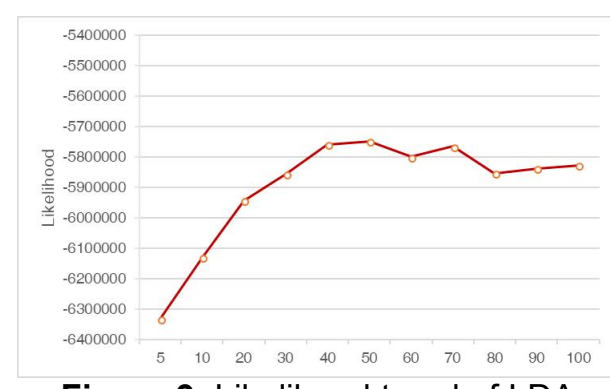

Figure 9. Likelihood trend of LDA

The name of each topic was determined according to the following strategies. First, referring to domain ontology, we group entities describe the same medical case, for example, 'heart' and 'surgery' would stand for the cardiac topic. Second, words with similar meaning or used to the relevant process were utilized for topic identification. For example, 'register', 'appointment' and 'check' account for the procedure that a patient uses e-Health service. Last, we decide the name of a topic based on the characteristic or property 
of a certain word. For example, 'careful', 'professional' and 'excellent' are usually used to describe the service quality of a doctor.

From Tables 5, we can directly see the apparent distinctiveness of several topics. Topic 1-3 are topics related to medication case while topic 4 represent service-related items. As for topic 5, it is noted as measures about a physician, for example, physician's service quality and medical literacy. The topic modelling result provide cue about patients' main focus or motivations when using online health consultation.

Table 5. Top 10 words and topic names of selected topic

\begin{tabular}{|c|c|c|c|c|}
\hline Topic 1: cardiopathy & $\begin{array}{c}\text { Topic 2: children got } \\
\text { a flu }\end{array}$ & Topic 3: eye disease & $\begin{array}{c}\text { Topic 4: online health } \\
\text { service }\end{array}$ & $\begin{array}{c}\text { Topic 5: service and } \\
\text { attitude of doctor }\end{array}$ \\
\hline patient & cough & child & check & responsible \\
\hline blood pressure & child & eyesight & attitude & leechcraft \\
\hline heart & baby & diagnosis & appointment & enthusiastic \\
\hline illness & asthma & shortsightedness & register & excellent \\
\hline symptom & kid & eye & hospital & kind \\
\hline care & flue & check & queue & attitude \\
\hline stent & allergy & astigmatism & expert & perspective \\
\hline diagnosis & diagnosis & glasses & professional & professional \\
\hline re-diagnosis & medicine & headache & responsible & careful \\
\hline electrocardiogram & pneumonia & patient & re-visit & quality \\
\hline
\end{tabular}

\section{Discussions and conclusions}

There have been many studies investigating the IT-Enabled health service. Previous research shows that the IT-Enabled collaborative technology plays an important role in enhancing the health service delivery, providing medical support and facilitate the collaboration among caregivers and health consumers [13]. However, few studies explored users' attitude to the ICT assisted health service. Our study filled this research gap through a case study analyzing user reviews posted on online health consultation website, via text analysis methods. Specifically, we leverage four natural language processing techniques, term frequency analysis, sentiment analysis, feature words clustering and topic modelling to explore how patients express their opinions on health services quality, physicians' attitude and website functionality. Our research further verified the popularity of IT-Enabled health service and provide a comprehensive interpretation of patients' WOM on online health consultation.

Overall, our analysis reveals some patterns of WOM on online health consultation: (1) From the results of the descriptive analysis, we can conclude that the IT-Enabled health service is experiencing an increased tendency as confirmed in other research [43]. (2) The results of the term frequency analysis show that while patients' concerns on IT-Enabled health service vary from doctors, website function, emotional support and the physician-patient relationship, the emphasis lies in service quality and benefit acquisition. (3) Sentiment analysis results show that most of the reviews carry a mild positive sentiment. Scarcely were reviews filled with extreme strong emotionality. This phenomenon is consistent in other IT-Enabled collaboration platforms [35]. In other words, most of the physicians seem reliable and responsible when providing online healthcare services. (4) We make conclusion from the result of the feature words clustering, that patients tend to mention their health conditions, social experience and emotions in WOM. It can be interpreted that health issue is usually related to individual's social environment and emotional status [47]. (5) Topic modelling results indicate that users are mostly concerned with physicians' medical skills and their attitude. As is refereed in previous research, patients are usually desired to be respected and supported by the doctor, apart from obtaining valuable services [22]. These findings are directions for the establishment and maintenance of the physician-patient relationship.

Concentrating on IT-Enabled collaborative health service, our research shows that present online health services are not good enough to satisfy public demands. As shown in sentiment analysis and topic modelling, many users may feel disappointed by unqualified service and unfair pricing. Despite the urgent needs of healthcare services delivered by ICT, the maturity of this industry is far from public expectations.

\section{Implications and future directions}


Our research results have both theoretical and practical implications in the construction of better collaboration in IT-Enabled healthcare service. First, our research extends the theoretical context in understanding the IT-Enabled health service from the perspective of users. Textual patterns contained in patients' reviews confirmed above assumptions and specifically shed light on how to better understand patients, enhance the quality of e-Health service and increase patient satisfaction during online health consultation. Through the emotional analysis of the posts in the forum, we can understand the emotional need of the OHC members. Second, our research provides numerous practical implications for implementing IT-Enabled collaboration project in healthcare service. For example, websites developers are supposed to optimize the user-interface and modify vulnerability. Doctors are suggested to establish an equal and relaxed relationship with patients, although sometimes there are a lot of orders waiting to be processed. More importantly, doctors should be aware of their responsibility for patients and be careful about each treatment. The manager of these website should check out their pricing and marketing strategies, take the responsibilities to figure out wrong information or fake information and make strict regulations to supervise the caregivers.

The current study also has several limitations that could be addressed in future research. First, the data we used are from only one online health community. It will be more persuasive if take more websites into account. Second, quantitative research checking the relationship between patients reviews and doctors' orders would deepen our comprehension of the impact of patient WOM. We are prepared to do further investigation on Internet healthcare industry.

\section{References}

[1] Ashurst, C., et al., "Exploring IT-enabled innovation: A new paradigm?", International Journal of Information Management, 2012. 32(4): p. 326-336.

[2] Atanasova, S., T. Kamin, and G. Petrï̈ , "Exploring the benefits and challenges of health professionalsâ€ ${ }^{\mathrm{TM}}$ participation in online health communities: Emergence of (dis) empowerment processes and outcomes", International Journal of Medical Informatics, 2017. 98: p. 13-21.

[3] Bengio, Y., et al., "A neural probabilistic language model", Journal of machine learning research, 2003. 3(Feb): p. 1137-1155.

[4] Blei, D.M., "Probabilistic topic models", Communications of the ACM, 2012. 55(4): p. 77-84.

[5] Blei, D.M., A.Y. Ng, and M.I. Jordan, "Latent dirichlet allocation", Journal of Machine Learning Research, 2003. 3(Jan): p. 993-1022.
[6] Bose, R., "Knowledge management-enabled health care management systems: capabilities, infrastructure, and decision-support", Expert Systems with Applications, 2003. 24(1): p. 59-71.

[7] Castellano, S. and V. Dutot, "Investigating the Influence of E-Word-of-Mouth on E-Reputation", International Studies of Management \& Organization, 2017. 47(1): p. 42-60.

[8] Chakravarty, A., Y. Liu, and T. Mazumdar, "The differential effects of online word-of-mouth and critics' reviews on pre-release movie evaluation", Journal of Interactive Marketing, 2010. 24(3): p. 185-197.

[9] Chen, H., et al., Knowledge management, data mining, and text mining in medical informatics, in Medical Informatics. 2005, Springer. p. 3-33.

[10] Chevalier, J.A. and D. Mayzlin, "The effect of word of mouth on sales: Online book reviews", Journal of marketing research, 2006. 43(3): p. 345-354.

[11] Dhaoui, C., C.M. Webster, and L.P. Tan, "Social media sentiment analysis: lexicon versus machine learning", Journal of Consumer Marketing, 2017. 34(6): p. 480-488.

[12] Effken, J.A. and P. Abbott, "Health IT-enabled care for underserved rural populations: The role of nursing", Journal of the American Medical Informatics Association, 2009. 16(4): p. 439-445.

[13] Eikey, E.V., M.C. Reddy, and C.E. Kuziemsky, "Examining the role of collaboration in studies of health information technologies in biomedical informatics: A systematic review of 25 years of research", Journal of Biomedical Informatics, 2015. 57: p. 263-277.

[14] Gao, R., et al. Developing simplified Chinese psychological linguistic analysis dictionary for microblog. in international conference on brain and health informatics. 2013. Springer.

[15] Greaves, F., et al., "Use of sentiment analysis for capturing patient experience from free-text comments posted online", Journal of Medical Internet Research, 2013. 15(11).

[16] Griffiths, T.L. and M. Steyvers, "Finding scientific topics", Proceedings of the National academy of Sciences, 2004. 101(suppl 1): p. 5228-5235.

[17] Harris, M.R., et al., "A term extraction tool for expanding content in the domain of functioning, disability, and health: proof of concept", Journal of Biomedical Informatics, 2003. 36(4-5): p. 250-259.

[18] Huh, J., et al. Patient moderator interaction in online health communities. in AMIA Annual Symposium Proceedings. 2013. American Medical Informatics Association.

[19] Ito, T.A., et al., "Negative information weighs more heavily on the brain: The negativity bias in evaluative categorizations", Journal of Personality and Social Psychology, 1998. 75(4): p. 887. 
[20] Johnston, A.C., et al., "Online health communities: An assessment of the influence of participation on patient empowerment outcomes", Information Technology \& People, 2013. 26(2): p. 213-235.

[21] Kamel Boulos, M.N. and S. Wheeler, "The emerging Web 2.0 social software: an enabling suite of sociable technologies in health and health care education 1", Health Information \& Libraries Journal, 2007. 24(1): p. 2-23.

[22] Kelley, J.M., et al., "The influence of the patient-clinician relationship on healthcare outcomes: a systematic review and meta-analysis of randomized controlled trials", PloS one, 2014. 9(4): p. e94207.

[23] Korkontzelos, I., et al., "Analysis of the effect of sentiment analysis on extracting adverse drug reactions from tweets and forum posts", Journal of Biomedical Informatics, 2016. 62: p. 148-158.

[24] Kvedar, J., M.J. Coye, and W. Everett, "Connected health: a review of technologies and strategies to improve patient care with telemedicine and telehealth", Health Affairs, 2014. 33(2): p. 194-199.

[25] Laouyane, A., Telemedicine and developing countries. 1998, SAGE Publications Sage UK: London, England.

[26] Larsen, K.R., et al., "Analyzing unstructured text data: Using latent categorization to identify intellectual communities in information systems", Decision Support Systems, 2008. 45(4): p. 884-896.

[27] Lee, M. and S. Youn, "Electronic word of mouth (eWOM) How eWOM platforms influence consumer product judgement", International Journal of Advertising, 2009. 28(3): p. 473-499.

[28] Litvin, S.W., R.E. Goldsmith, and B. Pan, "Electronic word-of-mouth in hospitality and tourism management", Tourism Management, 2008. 29(3): p. 458-468.

[29] Lu, H.-Y., B.R. Shaw, and D.H. Gustafson, "Online health consultation: Examining uses of an interactive cancer communication tool by low-income women with breast cancer", International Journal of Medical Informatics, 2011. 80(7): p. 518-528.

[30] $\mathrm{Lu}, \mathrm{N}$. and $\mathrm{H}$. Wu, "Exploring the impact of word-of-mouth about Physicians' service quality on patient choice based on online health communities", BMC Medical Informatics and Decision Making, 2016. 16(1): p. 151.

[31] Lu, S.F. and H. Rui, "Can We Trust Online Physician Ratings? Evidence from Cardiac Surgeons in Florida", Management Science, 2017.

[32] Moorhead, S.A., et al., "A new dimension of health care: systematic review of the uses, benefits, and limitations of social media for health communication", Journal of Medical Internet Research, 2013. 15(4). [33] Pai, R.R. and S. Alathur, "Assessing mobile health applications with twitter analytics", International
Journal of Medical Informatics, 2018. 113: p. 72-84. [34] Pang, B. and L. Lee, "Opinion mining and sentiment analysis", Foundations and TrendsÂ ${ }^{\circledR}$ in Information Retrieval, 2008. 2(1â€"2): p. 1-135.

[35] Qiu, L., J. Pang, and K.H. Lim, "Effects of conflicting aggregated rating on eWOM review credibility and diagnosticity: The moderating role of review valence", Decision Support Systems, 2012. 54(1): p. 631-643.

[36] Rahimi, B., V. Vimarlund, and T. Timpka, "Health information system implementation: a qualitative meta-analysis", Journal of Medical Systems, 2009. 33(5): p. 359.

[37] Riegner, C., "Word of mouth on the web: The impact of Web 2.0 on consumer purchase decisions", Journal of Advertising Research, 2007. 47(4): p. 436-447.

[38] Smyth, J.M., "Written emotional expression: effect sizes, outcome types, and moderating variables", Journal of Consulting and Clinical Psychology, 1998. 66(1): p. 174.

[39] Sparck Jones, K., "A statistical interpretation of term specificity and its application in retrieval", Journal of Documentation, 1972. 28(1): p. 11-21.

[40] Spink, A., et al., "A study of medical and health queries to web search engines", Health Information \& Libraries Journal, 2004. 21(1): p. 44-51.

[41] Sun, T., et al., "Online word-of-mouth (or mouse): An exploration of its antecedents and consequences", Journal of Computer-Mediated Communication, 2006. 11(4): p. 1104-1127.

[42] Swan, M., "Emerging patient-driven health care models: an examination of health social networks, consumer personalized medicine and quantified self-tracking", International Journal of Environmental Research and Public Health, 2009. 6(2): p. 492-525.

[43] Van De Belt, T.H., et al., "Definition of Health 2.0 and Medicine 2.0: a systematic review", Journal of Medical Internet Research, 2010. 12(2).

[44] Wicks, P., et al., "Sharing health data for better outcomes on PatientsLikeMe", Journal of Medical Internet Research, 2010. 12(2).

[45] Wu, H. and N. Lu, "Service provision, pricing, and patient satisfaction in online health communities", International Journal of Medical Informatics, 2018. 110.

[46] Wu, H.C., et al., "Interpreting tf-idf term weights as making relevance decisions", ACM Transactions on Information Systems (TOIS), 2008. 26(3): p. 13.

[47] Zapf, D., C. Knorz, and M. Kulla, "On the relationship between mobbing factors, and job content, social work environment, and health outcomes", European Journal of Work and Organizational psychology, 1996. 5(2): p. 215-237. 